\title{
Spatial soliton dynamics in cubic-quintic media
}

\author{
Nuno A. Silva ${ }^{\mathrm{a}, \mathrm{b}}$, M. I. Carvalho ${ }^{\mathrm{c}}$, A. Guerreiro ${ }^{\mathrm{a}, \mathrm{b}}$ \\ a INESC TEC - UOSE, R.Campo Alegre, 4169-007, Porto, Portugal. \\ b Faculdade de Ciências da Universidade do Porto, R.Campo Alegre, 4169-007, Porto, \\ Portugal. \\ c Faculdade de Engenharia da Universidade do Porto, R. Dr. Roberto Frias, 4200-465 Porto, \\ Portugal.
}

\begin{abstract}
In this paper we address soliton-soliton interactions in a nonlinear cubic-quintic optic media, using for that purpose numerical methods and high performance graphics processor unit (GPU) computing. We describe an implementation of GPU-based computational simulations of the generalized Nonlinear Schrodinger Equation, obtaining simulations more than 40 times faster relative to CPU-based simulations, especially in the multidimensional case. We focus our attention in the study of soliton collisions and scattering phenomena that, offering the possibility of steering light with light, open a path towards future optical devices.
\end{abstract}

Keywords: Solitons, Nonlinear Optics

\section{INTRODUCTION}

Exploring the dynamics of interacting solitons is a problem with major interest in nonlinear optics. Optical spatial solitons are robust and self-trapped light pulses that can propagate in nonlinear bulk media without changing their shape. In the presence of other solitons, ${ }^{1-4}$ linear refraction index ${ }^{5}$ or optical lattices of nonlinearities ${ }^{6,7}$ spatial solitons can display a wide range of behaviors, from wave-like and particle-like to liquid-like. These facts make solitons natural candidates for the transmission of data in communication systems ${ }^{8}$ and in optical processors, like ultra-fast logic gates. ${ }^{9}$ A material with competitive cubic-quintic nonlinearities can, support spatial solitons with more than one transverse dimensions. However, contrary to what happens in a cubic (Kerr) media which can be addressed using elegant analytical tools such Inverse Scattering Technique (IST), in cubic-quintic media this is not the case since the physical system is described by a non integrable equation. Because of this, it is preferable to use computational methods to study the dynamics of solitons in such systems. However, exploring large scale and multidimensional systems poses several challenges as the running times of the simulations are very large. It is then desirable to have new and more efficient tools which can speed up the computational simulations and make the research easier and more effective.

Parallel computing is currently one the most powerful tools to do computationally intensive calculations in both scientific research and engineering, and is becoming a common place available to almost anyone. Thanks to new programming frameworks like CUDA and OpenCL, it is now possible to use high performance heterogeneous platforms inside home desktops with many central and graphics processing units (CPUs and GPUs) to do scientific computation. Traditionally, graphical processing units are components specialized in image processing and are very fast in doing large amounts of certain types of calculations. However, in recent years the computer industry has put out new graphical processing platforms which contain hundreds and even thousands of cores packed in a single graphical board, working in parallel. This increases tremendously the computing power available, which can be used to address problems involving massive calculations if adequate control software is available. CUDA has been developed by NVIDIA specifically for general purpose use of graphics processing units (GPGPU) to do just this. The current version of CUDA includes several parallel numerical packages which simplify the development of a new generation of scientific codes.

In this paper we present our efforts in the design and implementation of a GPU based SSFM to solve the multidimensional generalized nonlinear Schrodinger equation (NLSE) for a cubic-quintic media. We show that this new methodology allows, not only to consider larger problems, but also, to solve them with an important 
speedup. As a case study, we address the study the collision of two 2-dimensional spatial solitons. In section 2 , we describe the physical model and the basic equations for spatial solitons described by the cubic and cubicquintic NLSE, presenting the analytical and approximate solutions for both case. In section 3, we describe the development of computational methods based on the Split Step Fourier Method (SSFM) to study the NLSE in nonlinear optical media. We also address the implementation of the code using either the CPU or the GPU, analyzing the speedups obtained in the later. In section 4, we present the numerical results for a system of colliding 2-dimensional spatial solitons. With the study of the soliton scattering in terms of the impact parameter and difference of global phase between the solitons, it is possible to identify different regimes of scattering and distinct soliton behavior, which includes not only wave-like but also particle-like and liquid-like behaviors. Finally, in section 5 we present the conclusions of this work.

\section{NLSE AND SOLITON SOLUTIONS}

The starting point of analysis is the generalized NLSE

$$
i \frac{\partial \psi}{\partial z}+\frac{1}{2} \nabla_{\perp}^{2} \psi+F\left(|\psi|^{2}\right) \psi=0
$$

which describes the evolution of the dimensionless amplitude of a light field $\psi$ in a nonlinear media with properties given by the function $F\left(|\psi|^{2}\right)$. Here $z$ is the longitudinal coordinate parallel to the propagation and $\nabla_{\perp}^{2}$ is the Laplacian in the transverse directions.

Media with a cubic nonlinearity (Kerr) characterized by $F\left(|\psi|^{2}\right)=|\psi|^{2}$ are a special case since equation (1) turns out to be integrable using the IST method, which allows to find soliton solutions of the form

$$
\psi(z, x)=2 \nu_{j} \operatorname{sech}\left\{2 \nu_{j}\left(x-\bar{x}_{j}\right)\right\} \exp \left\{i 2 \mu_{j}\left(x-\overline{x_{j}}\right)+i \delta_{j}\right\}
$$

where the parameters $\nu_{j}, \overline{x_{j}}, \mu_{j}$, and $\delta_{j}$ refer to the amplitude, the position, the frequency and the phase of the soliton.

Unfortunately, 2-d an 3-d spatial solitons are not stable in a Kerr media because (1) is usually non integrable. Nevertheless, it is assumed that the solutions of equation (1) can be described by a general soliton solution

$$
q(x, z)=A(z) g[B\{x-\bar{x}(z)\}] \exp (-i k(z)\{x-\bar{x}(z)\}+i \theta(z)),
$$

where $A, B, g, k, \theta, \bar{x}(t)$ are the amplitude, the width, the shape, the frequency, the phase and the center of the soliton, respectively. The existence and the dynamics of the soliton can be then be investigated using variational methods in terms of the variation of these parameters for specific forms of $F$, corresponding to different nonlinearities. Media with cubic-quintic nonlinearities are known to support solitons in more than one dimension and have been extensively studied because they are described by a simple nonlinear potential of the form $F\left(|\psi|^{2}\right)=|\psi|^{2}-\alpha|\psi|^{4}$. In this case, it is possible to obtain approximate solutions in the form of supergaussians pulses, ${ }^{10}$ defined by

$$
\psi(r, z)=A \exp \left[-B^{2}(r-\bar{r})^{2 m}\right] \exp (i \delta z)
$$

where $r$ is the vector with transverse coordinates and $m$ a parameter related to pulse energy. For the soliton to be stable, these parameters must be mutually related by the following conditions

$$
\begin{gathered}
A= \\
\delta=\frac{2 B^{2} m}{\Gamma(1+1 / m)} \frac{m+\ln (2 / 3)}{\ln (4 / 3)}
\end{gathered}
$$




$$
B=\left(\frac{1}{A} \sqrt{\frac{2^{1 / m}}{\Gamma(1+1 / m)} \frac{2 m-\ln 3}{\ln (4 / 3)}}\right)^{-1}
$$

As the system is non integrable, studying the dynamics of the solitons is only possible using either perturbative methods or numerical simulations. Here, we focus on the numerical simulations because they provide a more direct way of studying the interactions of solitons in wider range of situations.

\section{SSFM AND GPU IMPLEMENTATION}

The numerical method used is the extensively used $\mathrm{SSFM}^{11}$ for beam propagation. We start writing the generalized NLSE in the form

$$
\frac{\partial \psi}{\partial z}=(\hat{D}+\hat{N}) \psi
$$

with $\hat{D}=\frac{i}{2} \nabla_{\perp}^{2} \psi$ a linear operator relative to the dispersion and $\hat{N}=i F\left(|\psi|^{2}\right)$ relative to the nonlinearities of the media. From integration of equation (8) the exact solution is given by

$$
\psi(z+h, \vec{r})=\exp [h(\hat{D}+\hat{N})] \psi(z+h, \vec{r}),
$$

where $h$ is the step of discretization of variable $z$.

Assuming that for a small propagation distance $h$ the linear and nonlinear parts of the dynamics can be treated separately, a good approximation of the solution of equation (8) up to the second order of $h$ and with an error of order of $h^{3}$ is

$$
\psi(z+h, \vec{r}) \approx \exp \left(\frac{h}{2} \hat{D}\right) \exp (h \hat{N}) \exp \left(\frac{h}{2} \hat{D}\right) \psi(z, \vec{r}) .
$$

This means that computationally the solution $\psi(z+h, \vec{r})$ is calculated from $\psi(z, \vec{r})$ by applying sequentially the operators $\exp (h \hat{D} / 2), \exp (h \hat{N})$ and $\exp (h \hat{D} / 2)$ again.

The operator $\exp (h \hat{D} / 2)$ is a differential operator in the direct space but becomes algebraic in the Fourier space, which allows it to be quite easy and computationally inexpensive to calculate. Therefore, the idea is to evaluate the linear step in the Fourier space using

$$
\exp \left(\frac{h}{2} \hat{D}\right) \Psi(z, \vec{r})=F_{T}^{-1}\left\{\exp \left(-i h \frac{\vec{k} \cdot \vec{k}}{2}\right) F_{T}\{\Psi(z, \vec{r})\}\right\},
$$

where $F_{T}$ denotes the Fourier-transform operation and $\vec{k}$ is the wave number. Unlike the linear operator, the nonlinear operator $\exp (h \hat{N})$ must be calculated in the direct space where it is simple to calculate. This implies that during each step of the calculations, it is necessary to Fourier transform $\Psi(z, \vec{r})$, apply the operator $\exp (h \hat{D} / 2)$, reverse the Fourier transform on $\Psi(z, \vec{r})$, apply the nonlinear operator $\exp (h \hat{N})$, and so on and so forth. Fortunately, the computational cost of all these Fourier transforms is small because we use the fast Fourier transform algorithm, which is highly optimized.

Our computational code uses the equations (10) and (11) to solve numerically the NLSE. The code was developed in the $\mathrm{C}++$ programming environment and can use either the CPU or the GPU to do the calculations, depending on the choice of the user. The structure of the code is the same for both cases and only differs in the processor used. For the CPU, the Fourier transform is computed using the FFTW ${ }^{12}$ library and serial computations. The GPU analog uses the CUFFT ${ }^{13}$ for the Fourier transform and THRUST library for all other necessary operations, exploiting the power of parallel computations. The initial conditions, local or global 
1d NLSE

\begin{tabular}{|c|c|c|c|c|}
\hline \hline Number of time steps & $\mathrm{N}$ & GPU (s) & CPU (s) & Speedup \\
\hline \hline \multirow{3}{*}{250000} & $2^{9}$ & 3 & 21 & 7,0 \\
\cline { 2 - 5 } & $2^{10}$ & 4 & 45 & 11,3 \\
\cline { 2 - 5 } & $2^{11}$ & 5 & 100 & 20,0 \\
\cline { 2 - 5 } & $2^{12}$ & 9 & 180 & 20,0 \\
\cline { 2 - 5 } & $2^{13}$ & 12 & 375 & 31,3 \\
\hline
\end{tabular}

2d NLSE

\begin{tabular}{|c|c|c|c|c|}
\hline \hline Number of time steps & $\mathrm{NxN}$ & GPU (s) & CPU (s) & Speedup \\
\hline \multirow{4}{*}{1000} & $2^{7} \times 2^{7}$ & 1 & 34 & 34,0 \\
\cline { 2 - 5 } & $2^{8} \times 2^{8}$ & 3 & 137 & 45,7 \\
\cline { 2 - 5 } & $2^{9} \times 2^{9}$ & 15 & 569 & 37,9 \\
\cline { 2 - 5 } & $2^{10} \times 2^{10}$ & 61 & 2486 & 40,8 \\
\cline { 2 - 5 } & $2^{11} \times 2^{11}$ & 247 & 10744 & 43,5 \\
\hline
\end{tabular}

3d NLSE

\begin{tabular}{|c|c|c|c|c|}
\hline \hline Number of time steps & NxNxN & GPU (s) & CPU (s) & Speedup \\
\hline \multirow{3}{*}{1000} & $2^{6} \times 2^{6} \times 2^{4}$ & 6 & 266 & 44,3 \\
\cline { 2 - 5 } & $2^{6} \times 2^{6} \times 2^{5}$ & 13 & 532 & 40,9 \\
\cline { 2 - 5 } & $2^{6} \times 2^{6} \times 2^{6}$ & 25 & 1071 & 42,8 \\
\cline { 2 - 5 } & $2^{6} \times 2^{6} \times 2^{7}$ & 50 & 2188 & 43,8 \\
\hline
\end{tabular}

Table 1. Speedup results for NLSE in a cubic-quintic media. This table contains the running time for different simulations executed in cthe CPU and the GPU and the corresponding speedup factors. All simulations were done with $\Delta x=0.2$ and $h=0.01$ and using double precision.

nonlinearities, $h$ integration step, $\Delta x$ spatial discretization and $N$ total number of points are user-specified for each physical problem.

In our simulations we use a NVIDIA GeForce GTX 660TI GPU with 2GB RAM DDR5 and 915 CUDA cores and an INTEL core i7-3770K CPU with 16GB of RAM. In Table 1 we see that GPU simulations are up to 40 times faster than the CPU-based simulations. The results show also that the most significative speedups are obtained for 2-d and 3-d simulations. The fact that the price of the GPU and CPU is almost the same and not as expensive as a cluster of CPUs with same speedup results, shows that the GPU computation is a good option for obtaining faster simulations of soliton dynamics, especially in multidimensional systems.

\section{SCATTERING OF COLLIDING 2-DIMENSIONAL SPATIAL SOLITONS}

In this section we are interested in the computational analysis of two 2-dimensional solitons frontal collisions in the $x y$ plane. The spatial solitons dynamics are described by the cubic-quintic NLSE with $F\left(|\psi|^{2}\right)=|\psi|^{2}-\alpha|\psi|^{4}$. Choosing $\alpha=1$ and solitons with parameter $m=1$, the other parameters are set according to equations (4)-(7) all the other necessary parameters. In this study we initialize the solitons with a phase difference $\delta_{1}-\delta_{2}=0$ or $\pi$ and with opposing but equal velocities $\left|k_{1}\right|,\left|k_{2}\right|=k$, by multiplying the supergaussian shape (4) by $\exp (-i k(z)\{x-\bar{x}(z)\}+i \theta(z))$.

The global phase difference $\delta_{1}-\delta_{2}$ determines the nature of the interaction between the two solitons and ultimately their behavior, switching between particle, wave and liquid-like. As shown in, ${ }^{14}$ the phase difference between solitons determines whether the interaction is attractive or repulsive. In the situations considered here, the two solitons and their trajectories are completely symmetric (point reflective symmetry relative to the origin), therefore their phase difference and the character of their interaction remains constant throughout the simulations. Another determinant parameter in the soliton-soliton scattering is the angular momentum, which introduces an effective repulsion between the solitons, and is determined by the initial velocity $k$ and impact parameter $b$ of the solitons. For large impact parameters and low velocities, the interaction is very weak and both soliton almost are not deflected from a straight trajectory, being almost impossible to classify their behavior. 
Instead, for small impact parameters, the interaction is stronger and the wide variety of soliton behaviors can be observed.

For $\delta_{1}-\delta_{2}=\pi$, the two solitons are set to be out-of-phase and their interaction is strongly repulsive, as seen in figures 1a)-1o). This is confirmed in figure 2a), which shows the dependence of the scattering angle $\theta$ (measured as a deflection of the original straight trajectory) on the impact parameter. The increase of collision velocity further strengths the repulsive nature of the interaction but also forces the solitons to come closer to each other. As a result, in this limit the scattering angle approaches the results predicted by the scattering model for hard-spheres ${ }^{15}$ revealing the particle-like behavior of solitons.
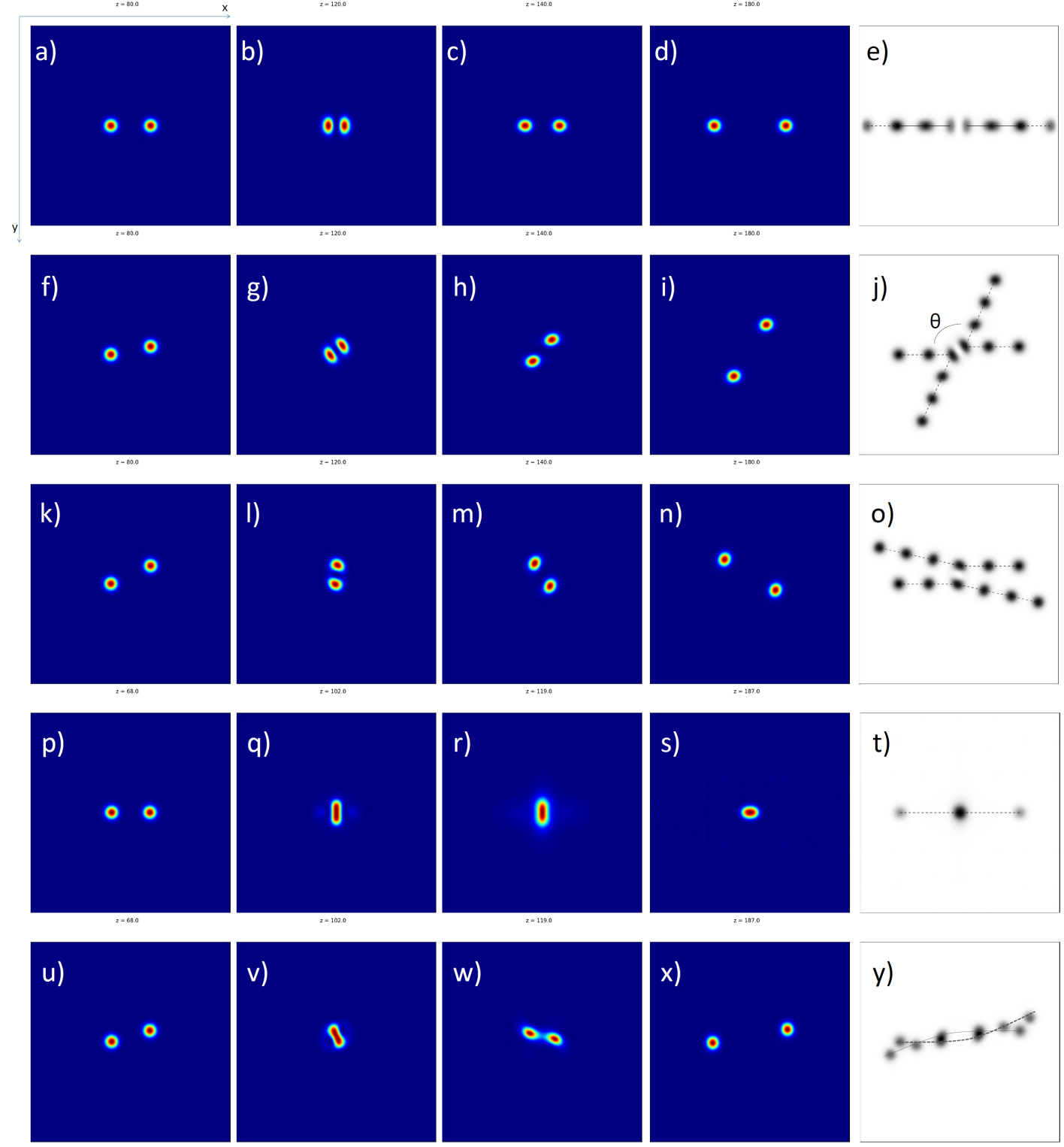

Figure 1. Typical numerical results for the evolution of two colliding solitons. Sequence 1a)-1e) shows a collision between two out-of-phase solitons with $k_{1}=0.2$ and $b=0$, sequence $\left.1 \mathrm{f}\right)-1 \mathrm{j}$ ) displays the results for $b=4$ and $1 \mathrm{k}$ )-1o) for $b=9$. Sequence 1p)-1t) displays the coalescence of two colliding in-phase solitons with $b=0$ and $k=0.3$. Sequence 1u)-1y) shows the results for $b=5$ and $k=0.3$.

For $\delta_{1}-\delta_{2}=0$, the two solitons are set to be in-phase and their interaction is attractive, as seen in figures 
1p) to 1y). However the anticipated particle-like dynamics does not hold for small impact parameter as the light pulses tend to coalesce, what reveals a liquid-like behavior of solitons. In figure 2 is seen that with the increase of the impact parameter the system undergoes a phase transition to a particle-like dynamics. For large impact parameters it is seen that the solitons almost do not interact, and thus the scattering angle is approximately null. With the decreasing of the impact parameter we see the expected increase of $\theta$. The peak in figure $2 \mathrm{~b}$ ) seems to be related with the formation of a metastable state with angular momentum, however further research is needed to understand the physics underlying this process.
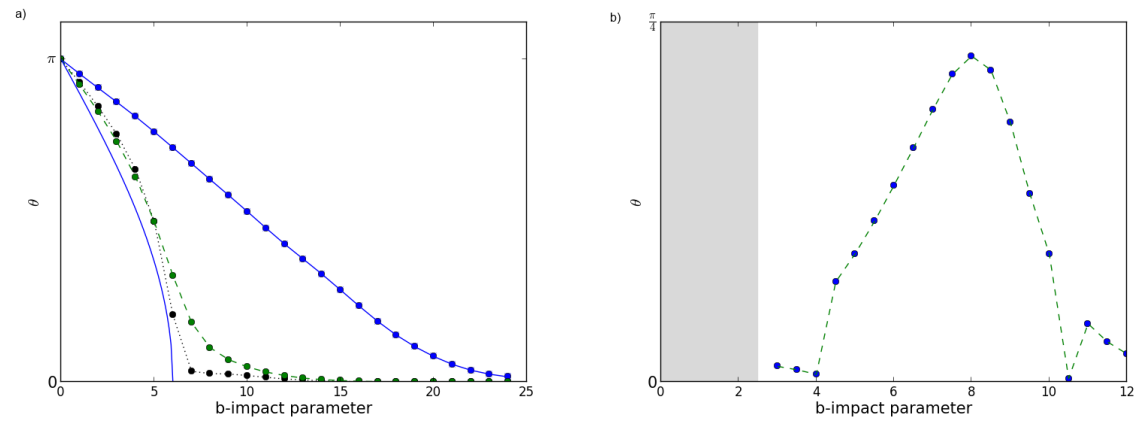

Figure 2. Computational results for the relation between the scattering angle and the impact parameter, $\theta(b)$. Figure a) displays the results for colliding out-of-phase solitons for $k=0.05$ (full line with markers), $k=0.2$ (dashed line with markers) and $k=0.3$ (pointed line with markers). The full line without markers shows the hard-sphere limit for a sphere with radius of 6 . Figure b) shows the results for colliding in-phase solitons with $k=0.3$. Shaded region is the zone of coalescence, where solitons reveal liquid-like behavior.

\section{CONCLUSIONS}

With this work we describe the implementation of a GPU-based code for the numerical simulation of NLSE, showing that CUDA enabled GPUs could reduce significantly the computation time of the simulation of the generalized NLSE, especially for multidimensional systems. Obtaining speedups of over 40, we present in this work a powerful tool that could be used not only in the research on the nonlinear optics field, but also in other physical systems described by nonlinear equations.

Taking advantaged from the significative speedups we have used the developed code to explore the dynamics of colliding 2-dimensional spatial solitons. We obtain two different regimes for the in-phase and out-of-phase solitons. While in the first case solitons reveal a particle-like behavior for a repulsive potential, the second one reveals both particle and liquid-like dynamics for attractive interaction potential. This type of dynamical studies may have potential applications in optical information processing and engineering.

\section{REFERENCES}

1. Aossey, D. W., Skinner, S. R., Cooney, J. L., Williams, J. E., Gavin, M. T., Andersen, D. R., and Lonngren, K. E., "Properties of soliton-soliton collisions," Phys. Rev. A 45, 2606-2610 (Feb 1992).

2. Kivshar, Y.S. and Malomed, B. A., "Dynamics of solitons in nearly integrable systems," Rev. Mod. Phys. 61, 763-915 (Oct 1989).

3. Yang, J. and Tan, Y., "Fractal structure in the collision of vector solitons," Phys. Rev. Lett. 85, 3624-3627 (Oct 2000).

4. Kivshar, Y. S., Agrawal, G. P., and Agrawal, G. P., [Optical Solitons], Academic Press, Burlington (2004).

5. Hasse, R. W., "Schrodinger solitons and kinks behave like newtonian particles," Phys. Rev. A 25, 583-584 (Jan 1982).

6. Kartashov, Y. V., Vysloukh, V. A., and Torner, L., "Solitons in complex optical lattices," The European Physical Journal Special Topics 173(1), 87-105 (2009).

7. Hang, C. and Konotop, V. V., "Spatial solitons in a three-level atomic medium supported by a laguerregaussian control beam," Phys. Rev. A 83, 053845 (May 2011). 
8. Haus, H. A. and Wong, W. S., "Solitons in optical communications," Rev. Mod. Phys. 68, 423-444 (Apr 1996).

9. McLeod, R., Wagner, K., and Blair, S., "(3+1)-dimensional optical soliton dragging logic," Phys. Rev. A 52, 3254-3278 (Oct 1995).

10. Dimitrevski, K., Reimhult, E., Svensson, E., Ohgren, A., Anderson, D., Berntson, A., Lisak, M., and Quiroga-Teixeiro, M. L., "Analysis of stable self-trapping of laser beams in cubic-quintic nonlinear media," Physics Letters A 248(5-6), 369-376 (1998).

11. Taha, T. and Xu, X., "Parallel split-step fourier methods for the coupled nonlinear schrodinger type equations," The Journal of Supercomputing 32(1), 5-23 (2005).

12. Frigo, M. and Johnson, S. G., "The Design and Implementation of FFTW3," Proceedings of the IEEE 93, 216-231 (Feb. 2005).

13. Nvidia, "CUDA CUFFT Library," (2007).

14. Atai, J., "Interaction of bragg grating solitons in a cubic-quintic medium," Journal of Optics B: Quantum and Semiclassical Optics 6(5), S177 (2004).

15. Kibble, T. W. B. and Berkshirel, F. H., [Classical Mechanics], Imperial College Press, London, fifth ed. (2004). 